\title{
Global transcript and phenotypic analysis of yeast cells expressing Ssa1, Ssa2, Ssa3 or Ssa4 as sole source of cytosolic Hsp70-Ssa chaperone activity
}

Naushaba Hasin ${ }^{1,2}$, Sarah A Cusack', Shahin S Ali ${ }^{3}$, David A Fitzpatrick ${ }^{4}$ and Gary W Jones ${ }^{1 *}$

\begin{abstract}
Background: Cytosolic Hsp70 is a ubiquitous molecular chaperone that is involved in responding to a variety of cellular stresses. A major function of Hsp70 is to prevent the aggregation of denatured proteins by binding to exposed hydrophobic regions and preventing the accumulation of amorphous aggregates. To gain further insight into the functional redundancy and specialisation of the highly homologous yeast Hsp70-Ssa family we expressed each of the individual Ssa proteins as the sole source of Hsp70 in the cell and assessed phenotypic differences in prion propagation and stress resistance. Additionally we also analysed the global gene expression patterns in yeast strains expressing individual Ssa proteins, using microarray and RT-qPCR analysis.

Results: We confirm and extend previous studies demonstrating that cells expressing different Hsp70-Ssa isoforms vary in their ability to propagate the yeast $\left[\mathrm{PSI}^{+}\right]$prion, with Ssa3 being the most proficient. Of the four Ssa family members the heat inducible isoforms are more proficient in acquiring thermotolerance and we show a greater requirement than was previously thought, for cellular processes in addition to the traditional Hsp104 protein disaggregase machinery, in acquiring such thermotolerance. Cells expressing different Hsp70-Ssa isoforms also display differences in phenotypic response to exposure to cell wall damaging and oxidative stress agents, again with the heat inducible isoforms providing better protection than constitutive isoforms. We assessed global transcriptome profiles for cells expressing individual Hsp70-Ssa isoforms as the sole source of cytosolic Hsp70, and identified a significant difference in cellular gene expression between these strains. Differences in gene expression profiles provide a rationale for some phenotypic differences we observed in this study. We also demonstrate a high degree of correlation between microarray data and RT-qPCR analysis for a selection of genes.

Conclusions: The Hsp70-Ssa family provide both redundant and variant-specific functions within the yeast cell. Yeast cells expressing individual members of the Hsp70-Ssa family as the sole source of Ssa protein display differences in global gene expression profiles. These changes in global gene expression may contribute significantly to the phenotypic differences observed between the Hsp70-Ssa family members.
\end{abstract}

Keywords: Saccharomyces cerevisiae, Prion, Heat shock, Stress, Hsp70, Ssa1, Ssa2, Ssa3, Ssa4, Chaperone, Gene expression

\footnotetext{
* Correspondence: gary.jones@nuim.ie

${ }^{1}$ Yeast Genetics Laboratory, Department of Biology, National University of

Ireland Maynooth, Maynooth, County Kildare, Ireland

Full list of author information is available at the end of the article
} 


\section{Background}

A major class of heat shock proteins (Hsps) belong to the ubiquitous Hsp70 family, a diverse collection of $70 \mathrm{kDa}$ chaperones that exist in various cellular compartments. Hsp70s perform essential housekeeping functions in protein folding, protein synthesis, translocation across membranes, protein degradation, assembly and disassembly of macromolecular complexes or aggregates, gene induction and apoptosis [1-4]. They are also involved in quality control process such as protein refolding after stress and control the activity of regulatory proteins in signal transduction pathways [5]. All of these cellular activities of Hsp70 depend upon its ability to interact with hydrophobic stretches of proteins in an ATP-dependent manner preventing non-productive interactions that would lead to aggregation and to promote protein refolding [6]. Hsp70 constitutes one of the most highly conserved and well-studied Hsps across species ranging from archaebacteria to plants and humans with the prokaryotic Hsp70 protein DnaK sharing approximately $50 \%$ amino acid similarity with eukaryotic Hsp70 proteins [7-10]. Various inter species expression studies of Hsp70 showed its conserved functional properties across distant species [11-15].

It had been suggested for a long time that the Hsp70 isoforms are functionally redundant and differ only by their spatio-temporal expression pattern. However, this was challenged by several findings in Saccharomyces cerevisiae (yeast) and higher eukaryotes demonstrating some functional specificity among Hsp70 isoforms [16,17]. In yeast, the $h s p 70$ gene family comprises of fourteen genes, whose protein products share a sequence similarity of approximately $50-96 \%$. Of these, nine are cytosolic and five are compartmental specific. The major cytosolic Hsp70 family in yeast is the Hsp70-Ssa (Stress Seventy sub-family A), which consists of four members of Ssa (Ssa1-4). These four isoforms are functionally redundant to some degree as expression of at least one family member is essential for growth [18]. Though other cytosolic Hsp70 sub-families cannot substitute for the survival function of Ssa subfamilies, the four Ssa proteins can compensate for each other $[19,20]$. Constitutively expressed Ssa1 and Ssa2 are 97\% identical to each other and under optimal conditions Ssa2 is approximately fourfold more abundant than Ssa1 and depletion of Ssa2 induces expression of Ssa1, maintaining overall Hsp70 abundance. The heat-inducible Ssa3 and Ssa 4 are $87 \%$ identical to each other and share an identity of $80 \%$ with Ssa1/2 [21]. The heat inducible isoforms are expressed under non-optimal growth conditions and help protect cells from the adverse effects of stress [22]. A major functional distinction between Ssa1 and Ssa2 exists in their effects on yeast prion propagation. The yeast $\left[\mathrm{PSI}^{+}\right]$ and [URE3] prions are infectious forms of the Sup35 (involved in translation termination) and Ure2 (involved in regulating usage of poor nitrogen sources) proteins respectively [23]. Chaperones of various families, including Hsp70s, have been shown to play an integral part in modulating prion propagation [24,25]. Overexpression of Ssa1 but not Ssa2, can cure [URE3] while depleting Ssa1 weakens $\left[\mathrm{PSI}^{+}\right]$but not $[U R E 3][26,27]$. The functional difference in Ssa1 or 2 in terms of prion propagation were found to be due to a single amino acid difference in the ATPase of these highly homologous proteins [28]. Hsp70 has also been implicated in biofilm production in yeast, which is another good example of functionally specificity among the Hsp70 isoforms [29]. Deletion of ssa1 had a more adverse effect on biofilm formation in yeast compared to ssa2 deletion. Additionally, Ssa3 and Ssa4 deletion enhanced the defects brought about by Ssa1/Ssa2 deletion suggesting cooperation between constitutive and inducible isoforms of Hsp70 [29]. Recently it was shown that Ssa1 (and probably other Ssa proteins) act as signal transducers mediating growth control through G1 cyclin abundance and activity, a process dependent on Ssa phosphorylation status at a highly conserved threonine residue in the ATPase domain [30]. Given the importance of the Hsp70 family in essential cellular functions and also that Hsp70 is a potential therapeutic target for a variety of human diseases, it is important to understand Hsp70s essential and non-essential roles within the cell and to characterize the functional difference between members of this chaperone family.

Here we make use of the genetically tractable yeast system. Using the plasmid shuffle technique we constructed yeast strains expressing either Ssa1, 2, 3 or 4 as the sole source of Hsp70-Ssa protein in the cell. To provide new insights into functional conservation and redundancy in the Hsp70-Ssa family, we carried out a comparative phenotypic analysis of these strains coupled with a global transcriptome analysis.

\section{Methods}

\section{Strains, Plasmids and Genetic methods}

The yeast strain used in this study was G402 (MATa ade2-1 SUQ5 kar1-1 his3 leu2 lys2 trp1 ura3 ssa1:: KanMX, ssa2::HIS3, ssa3::TRP1, ssa4::URA3-1f/pRDW10 [31]. All media used were as previously described by Loovers et al. [32]. Cultures were grown at $30^{\circ} \mathrm{C}$ unless indicated otherwise.

Plasmid pRDW10 contained in G402 strain [33] is a URA3-based, low-copy number centromeric plasmid with Ssa1, Ssa2, Ssa3 or Ssa4 as the sole source of Ssa in the cell under control of the SSA2 promoter. The SSA2 promoter was chosen as it is the only truly constitutive SSA promoter and allows comparative assessment of $S S A$ gene and protein function without complicating factors such as heat shock induction. The LEU2-based plasmids pC210 (= pC210-Ssa1), pDCM62 (= pC210-Ssa2), 
pA3 (= pC210-Ssa3) and pA4 (= pC210-Ssa4) were described by Sharma et al. and Schwimmer and Masison $[22,26]$. Plasmid pDCM90 is a URA3-based low-copy plasmid containing a gene for expression of a thermolabile bacterial luciferase LuxAB [34] on a ClaI-SmaI fragment. The pRS series of plasmids have been previously described by Sikorski et al. [35].

Monitoring of $\left[\mathrm{PSI}^{+}\right]$was carried out as described by Jones et al. [31]. Briefly, the presence of $\left[P S I^{+}\right]$and the weak suppressor tRNA SUQ5 in the strains were monitored as producing white colonies on media containing limiting amounts of adeneine, this is due to partial suppression and translation read through of the aberrant stop codon in the ade2.1 allele [36,37]. Nonsuppressed ade2-1 mutants are adenine auxotrophs and are red when grown on adenine limiting media.

\section{Construction of G402 expressing only one Hsp70-Ssa family member}

The plasmid shuffle technique was employed as essentially described by Loovers et al. [32] with minor modifications. The G402 strain contains the plasmid pRDW10, which contains a $U R A 3$ marker and is the sole source of Ssa in the strain. G402 was transformed with a LEU2 plasmid expressing either SSA1, 2, 3 or 4. Transformation plates were replica plated onto selective medium containing 5-fluoroorotic acid (5-FOA), a chemical that selects against URA3 cells and hence against the presence of the pRDW10 plasmid. After 3 days at $30^{\circ} \mathrm{C}$ incubation, colonies were purified on medium lacking leucine and confirmed as uracil auxotrophs.

\section{Acquired thermotolerance Assay}

Acquired thermotolerance assays were performed as described by Jung et al. [38] with minor modifications. Briefly, exponentially growing cultures were aliquoted and transferred to ice before exposing them to a temperature of $39^{\circ} \mathrm{C}$ for 1 hour to induce Hsp104. Subsequently, $1 \mathrm{ml}$ volumes of cell aliquots were maintained in a $47^{\circ} \mathrm{C}$ shaking incubator and transferred to ice at the indicated time points. Serial dilution of the aliquots was carried out and the cells were spotted onto appropriate agar plates and incubated for 3 days at $30^{\circ} \mathrm{C}$ and growth were monitored over the period of time. The viability at time zero was set to $100 \%$.

\section{Luciferase Assay}

Luciferase refolding was assayed as essentially described by Parsell et al. [34]. Overnight cultures were diluted to an $\mathrm{OD}_{600 \mathrm{~nm}}=0.2$ into the same selective medium and incubated at $37^{\circ} \mathrm{C}$ shaking for 30 minutes to induce expression of heat shock proteins. Subsquently, cellular luciferase activity of each strain was measured immediately using a FB 12 Luminometer (Berthold Detection Systems) following addition of $10 \mu \mathrm{l}$ decanal, and the cells were transferred to $45^{\circ} \mathrm{C}$ with shaking for 1 hour. Cyclohexamide was added at a concentration of $10 \mu \mathrm{g} / \mathrm{ml}$ after 50 minutes at $45^{\circ} \mathrm{C}$ to prevent de novo synthesis of luciferase during the recovery period. Cultures were then further incubated at $45^{\circ} \mathrm{C}$ for 10 minutes. Cultures were shifted to $25^{\circ} \mathrm{C}$ for the recovery of cells from heat shock. Thereafter, luciferase refolding activity was measured as described above at 15 minute time intervals (for 1-hour) to check for luciferase recovery.

\section{Guanidine Curing}

Routine curing of $\left[\mathrm{PSI}^{+}\right]$strains was done by streaking the yeast strains on YPD plates containing $3 \mathrm{mM} \mathrm{Gdn-HCl}$ and incubating the plates at $30^{\circ} \mathrm{C}$ for 3 days as essentially described by Jung et al. [33]. Cells were re-streaked onto YPD and red colonies were isolated. Both $\left[\mathrm{PSI}^{+}\right]$and $\left[p s i^{-}\right]$ versions of the strains were maintained at $4^{\circ} \mathrm{C}$ and the stock was stored at $-70^{\circ} \mathrm{C}$.

\section{Western analysis}

Western analysis was performed as described by Moran et al. [39]. Hsp70 monoclonal antibody was purchased from Cambridge Biosciences (Cambridge, UK) (SPA 822) and Hsp104 polyclonal antibody was a gift from John Glover (University of Toronto). Antibodies specific for Ssa1/2 and Ssa3/4 were a gift from Elizabeth Craig (University of Wisconsin, USA).

\section{RNA extraction}

Total RNA was extracted from $5-\mathrm{ml}$ cultures of the [ $\left.p s i^{-}\right]$ yeast strains expressing either Ssa1, Ssa2, Ssa3 or Ssa4 grown overnight at $30^{\circ} \mathrm{C}$. RNA was extracted using the Qiagen RNAesy kit as per manufacturer's recommendation. For each strain, three replicates were used and each experiment was conducted twice. RNA was DNasedtreated using TURBO DNA-free kit (Ambion, USA), according to the manufacturers recommendations. RNA concentrations were measured using a NanoDrop 1000 Spectrophotometer.

\section{Microarray analysis}

For microarray analysis, equal amount of RNA from all three replicates from two separate experiments were pooled together and sent to Toray Industries, Japan. 3DGene $^{\mathrm{TM}}$ Yeast Oligo chip S.cerevisiae $6 \mathrm{k}$ was used for the microarray analysis. The microarray analysis was done according to the manufacturer's instructions. In brief, aRNA (amino allyl-labeled RNA) was synthesized from $1 \mu \mathrm{g}$ total RNA with Amino Allyl MessageAmp ${ }^{\text {TM }}$ II aRNA Amplification kit (\#1753: Ambion). $10 \mu \mathrm{g}$ of aRNA was labelled with Cy5 Mono-ReactiveDyePack (PA25001:GE Health Care Bioscience) and $1 \mu \mathrm{g}$ of labelled aRNA was hybridized at $37^{\circ} \mathrm{C}(250 \mathrm{rpm})$ for 16 hours. The washed and dried DNA chip was scanned by ScanArray Lite (Perkin Elmer). The obtained microarray images were quantified 
using GenePix Pro6.0 and the spot intensity was calculated by taking the median intensity of the foreground signals. The background signal intensity is derived by taking the mean signal intensity of the blank spots that excludes the top and bottom 5\% signal intensities. The detected spots were defined as those that had signal intensity above the $95 \%$ upper confidence interval of the background signal intensity. For detected spots, their signal intensities were determined after subtracting with the mean background signal. For data comparison, the backgroundsubtracted signal intensity was normalized using global normalization in which the median from each microarray was used. Global normalize values were LOG-transformed to linearize the data and a heat map was created using TIGR MultiExperiment Viewer (MeV) [40]. All microarray data from this study are complaint with Minimum Information About a Microarray Experiment (MIAME) and is publicly available through the NCBI's Gene Expression Omnibus (GEO) database (http://www.ncbi.nlm.nih. gov/geo) under the series record GSE32433.

\section{Real time RT-PCR analysis}

Real-time RT-PCR analysis was used to verify the microarray results. Reverse transcription (RT) of total RNA was conducted as described by Ali et al. [41]. RT products were diluted to $200 \mu \mathrm{l}$ and $2.5 \mu \mathrm{l}$ were PCR-amplified in a $25 \mu \mathrm{l}$ volume reaction containing $12.5 \mu \mathrm{l}$ Premix Ex Taq ${ }^{\mathrm{TM}}$ (Perfect Real Time) (Takara, Japan) and $100 \mathrm{nM}$ each of forward and reverse transcript-specific primers (See Additional file 1: Table $\mathrm{S} 1$ ). PCR reactions were conducted in a Stratagene $\mathrm{M} \times 3000^{\mathrm{TM}}$ real-time PCR machine (Stratagene, USA) and the programme consisted of 1 cycle of $95^{\circ} \mathrm{C}$ for 10 seconds, 40 cycles of $95^{\circ} \mathrm{C}$ for 5 seconds, $60^{\circ} \mathrm{C}$ for 30 seconds (annealing and polymerization) and 1 further cycle of $95^{\circ} \mathrm{C}$ for $60 \mathrm{sec}-$ onds prior to melting curve analysis. Data were analysed using Stratagene $\mathrm{M} \times 3000^{\mathrm{TM}}$ software (Stratagene, USA). All the 24 RT samples along with their minusRT product amplified for the $S$. cerevisiae ACT1 (YFL039C) gene and average threshold cycle (CT) for RT samples was $19.23 \pm 0.17$ while CT value for the minus-RT samples were $\leq 37.29$. The expression patterns of the ACT1 gene was used to normalise the RTPCR data and the real-time quantification of target and housekeeping transcript accumulation was performed in separate reactions. The $\mathrm{CT}$ values obtained by realtime PCR (RT-qPCR) were used to calculate the accumulation of target gene (relative mRNA accumulation), relative to $A C T 1$ transcript, by $2^{\wedge-\Delta \Delta C t}$ method, where $\Delta \Delta \mathrm{Ct}=(\mathrm{Ct}$, Target gene - Ct, ACT1) [42]. For each strain, three replicate samples were used and each experiment was conducted twice. The relative mRNA accumulation were LOG-transformed to linearize the data and a heat map of was created using TIGR MultiExperiment Viewer (MeV) [40].

\section{Statistical analysis}

Normal distributon of data set was determined using the Ryan Joiner test [43] within Minitab (Minitab release $13.32^{\odot}, 2000$ Minitab Inc.). Non-normally distributed data were transformed to fit a normal distribution using the Johnson transformation [44] within Minitab (Minitab release $13.32^{\odot}, 2000$ Minitab Inc.). The homogeneity of datasets across replicate experiments was confirmed by one-tailed correlation analysis conducted using mean data values (non-normal data: Spearman Rank; normal data: Pearson product moment) conducted within the Statistical Package for the Social Sciences (SPSS 11.0, SPSS Inc.) $(r \geq 0.610 ; P<0.050)$ [45]. Therefore, datasets from the replicate experiments were pooled for the purposes of further statistical analysis. The significance of treatment effects was analysed using SPSS by either (i) normally distributed data - one-way ANOVA with Post Hoc pair wise Least Significance Difference (LSD) comparisons $(P=0.050)$, or (ii) non-normally-distributed data - the Kruskal-Wallis $\mathrm{H}$ test. Correlations between mean values from different normally distributed datasets were calculated using Pearson product moment analysis.

\section{Results}

\section{Confirmation of individual Hsp70-Ssa expression and prion propagation phenotype in strain G402}

To assess the functions of individual members of the Ssa family, each member was expressed as a sole source of Ssa in the G402 strain using the plasmid shuffle technique. To confirm the identity of the strains constructed we verified Ssa chaperone protein expression and Hsp104 expression levels using Western blotting (Additional file 2: Figure S1) and prion propagation phenotype (Figure 1). As reported previously by Sharma et al. [21] the members of the Ssa family individually supported the growth of G402 (Figure 1). The $\left[\mathrm{PSI}^{+}\right]$strain was well maintained in cells expressing Ssa1 and particularly Ssa3 as the sole source of Ssa, as seen by the pigmentation on YPD and growth on adenine lacking media (Figure 1). The prion phenotype was also maintained in cells expressing Ssa2 but not to the same extent as Ssa1 or 3, while cells expressing only Ssa4 were seen to impair the propagation of $\left[\mathrm{PSI}^{+}\right]$the most dramatically (Figure 1), these findings are in agreement with Sharma and Masison [22]. Following plasmid shuffle all constructed strains were confirmed as $\left[\mathrm{PSI}^{+}\right]$by mating analysis and curing with $\mathrm{Gdn}-\mathrm{HCl}$. All Ssa family members were well expressed from the Ssa2 promoter and no major changes are seen in the expression levels of Hsp104, except perhaps for a minor increase when Ssa3 is the sole Ssa expressed (Additional file 2: Figure S1). 




There are no major changes in Ssa or Hsp104 expression levels in $\left[\mathrm{PSI}^{+}\right]$or $\left[\mathrm{psi}^{-}\right]$variants of strains used in this study (Additional file 3: Figure S2). Using well-established plate assays the curability of $\left[\mathrm{PSI}^{+}\right]$appeared similar for cells expressing different Hsp70-Ssas to the prion-curing agents $\mathrm{Gdn}-\mathrm{HCl}$, 6-aminophenanthridine and guanabenz acetate [46-48].

\section{Acquired thermotolerance activity of the Ssa family}

The survival rate of yeast post-lethal heat shock can be greatly improved by pre-treating cells with mild nonlethal heat shock, which stimulates elevation of heat shock proteins, including Hsp70 but primarily Hsp104 $[8,49]$. Previously it had been demonstrated that yeast cells expressing only Ssa3 or Ssa4 (inducible Ssas) were more heat tolerant than those expressing only Ssa1 or Ssa2 (constitutive Ssas). It was suggested that this could be due to more efficient cooperation between inducible Hsp70s with Hsp104 compared to constitutive Hsp70s [21]. To address this question we carried out an acquired thermotolerance assay where the induced levels of Hsp104 expression provide resistance to prolonged exposure at high temperature. To assess the possible influence of the prion on survival, we assessed both $\left[\mathrm{PSI}^{+}\right]$ and $\left[p s i^{-}\right]$variants. Using this assay we find that both Ssa3 and Ssa4 show enhanced acquired thermotolerance compared to Ssa1 and Ssa2, and this difference is much more pronounced in $\left[\mathrm{psi}^{-}\right]$variants (Figure 2, left panels). We assessed the contribution of Hsp104 to the enhanced thermotolerance of the inducible Hsp70s by plating surviving cells on medium containing $3 \mathrm{mM}$ $\mathrm{Gdn}-\mathrm{HCl}$ (Figure 2, right panels). Gdn- $\mathrm{HCl}$ inhibits in vivo activity of Hsp104 [38,50]. Acquired thermotolerance was reduced for all strains compared to YPD medium, but the impact of Hsp104 inhibition was much less reduced for cells expressing Ssa3 as the sole Hsp70Ssa. This suggests that Ssa3 has a more significant role in Hsp104-independent acquired thermotolerance compared to other Ssa proteins or perhaps there are some other differences for Ssa3 in terms of global transcriptomic or proteomic responses to heat shock or stress compared to other Ssas.

\section{In vivo protein refolding ability of individual Hsp70-Ssas}

We next assessed whether the in vivo protein refolding activity of the individual Ssas reflected the results from the acquired thermotolerance plate assays. We used the in vivo reactivation of thermo-labile bacterial luciferase as a measure of protein refolding ability of Hsp70-Ssa chaperones (Figure 3). In contrast to the thermotolerance plate assays, Ssa1 expressing cells were the most efficient at reactivating denatured luciferase with Ssa3

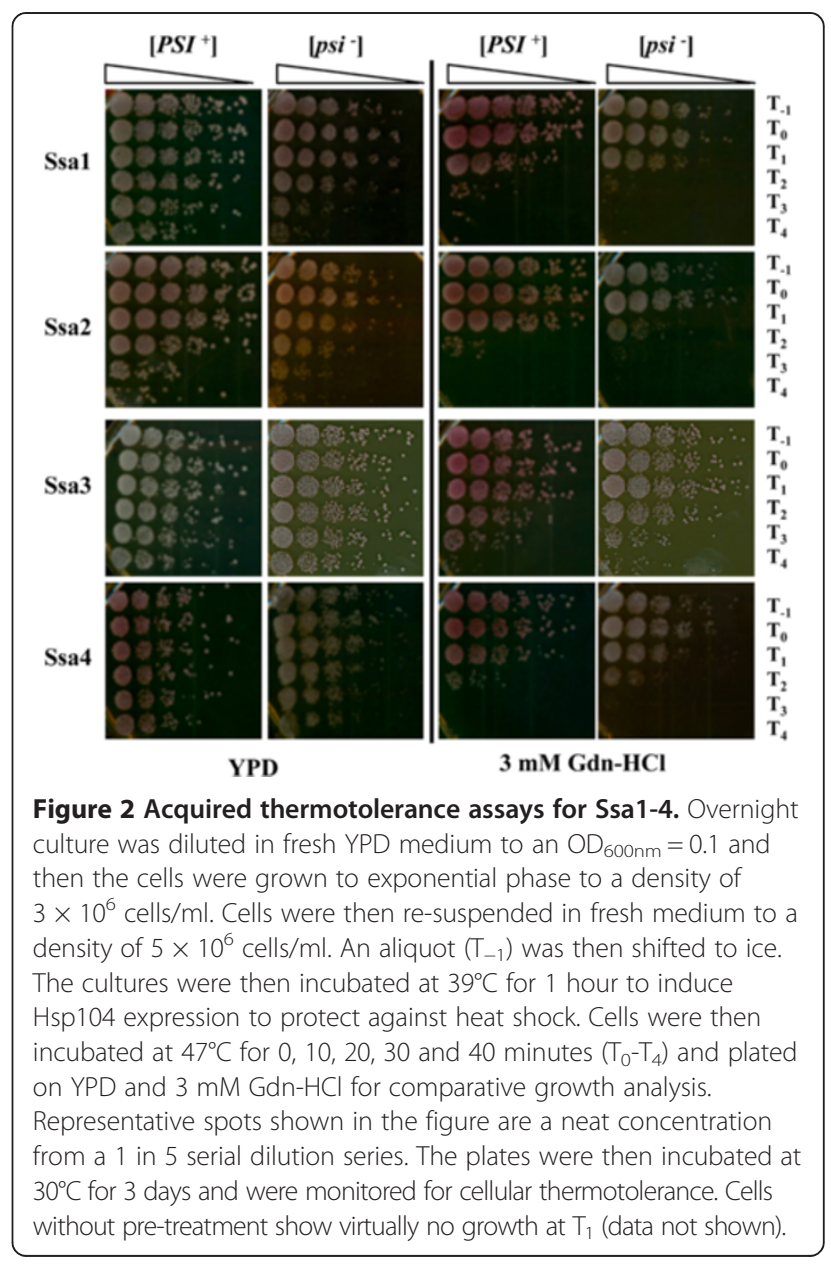


eventually reaching comparable levels with Ssa1 during the time course of the experiment. Ssa2 and Ssa4 showed similar activity but both reactivating approximately $20 \%$ less luciferase than Ssa1 and Ssa3 at the 45-minute time point. We also observed that the $\left[\mathrm{PSI}^{+}\right]$variants of Ssa1 and $\mathrm{Ssa} 3$ were more efficient at luciferase reactivation compared to $\left[p s i^{-}\right]$variants (Figure 3 ). The reason for this difference is unknown, but is unlikely to be due to alterations in chaperone levels (Additional file 2: Figure S1). As with the acquired thermotolerance plate assay, the presence of $\mathrm{Gdn}-\mathrm{HCl}$ in cultures prior to carrying out the assay causes a major reduction in luciferase refolding activity (due to inhibition of Hsp104, data not shown).

\section{Differences in other stress phenotypes exhibited by the Ssa family}

In addition to differences in heat shock phenotypes of cells expressing different Ssa family members, we also observed differences in responses to the cell wall damaging agent, sodium dodecyl sulfate (SDS) (Figure 4) and the oxidative stress inducer, hydrogen peroxide $\left(\mathrm{H}_{2} \mathrm{O}_{2}\right)$ (Figure 5).

Cells expressing Ssa4 exhibited greater resistance than other Ssas to SDS and the presence of $\left[\mathrm{PSI}^{+}\right]$also had an influence on SDS sensitivity for Ssa2 and Ssa3 expressing

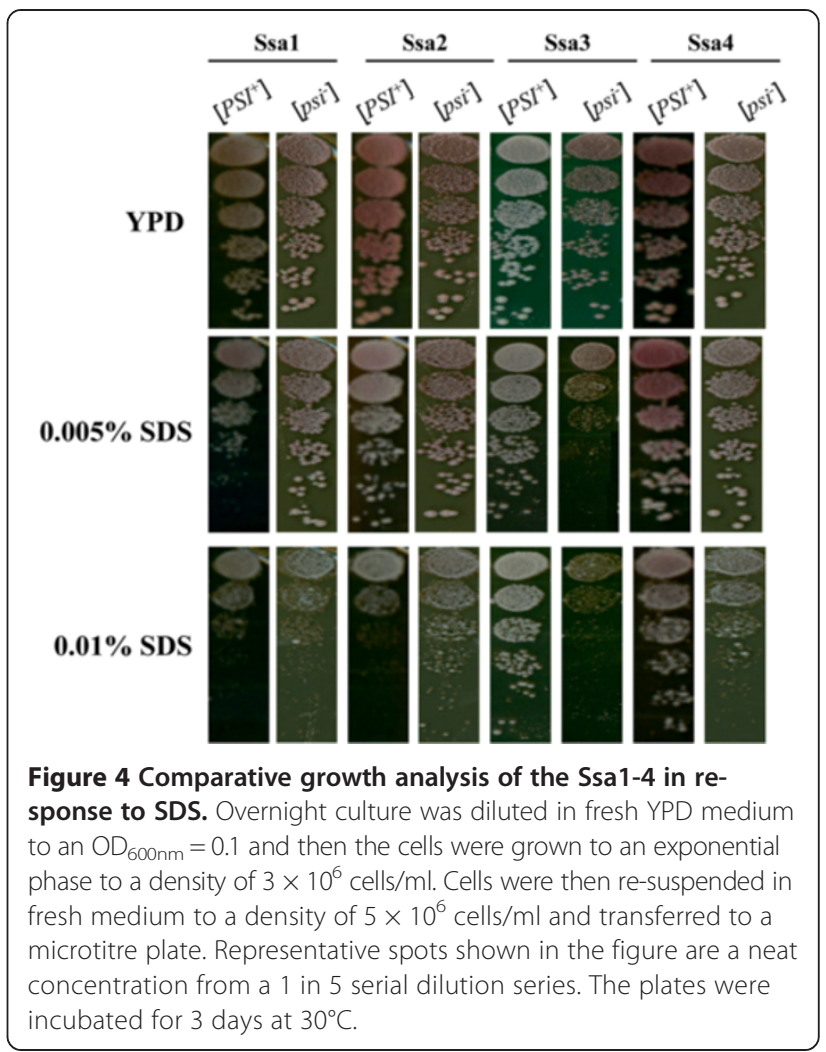

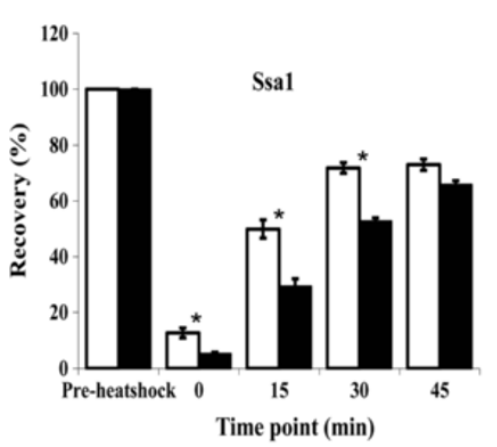
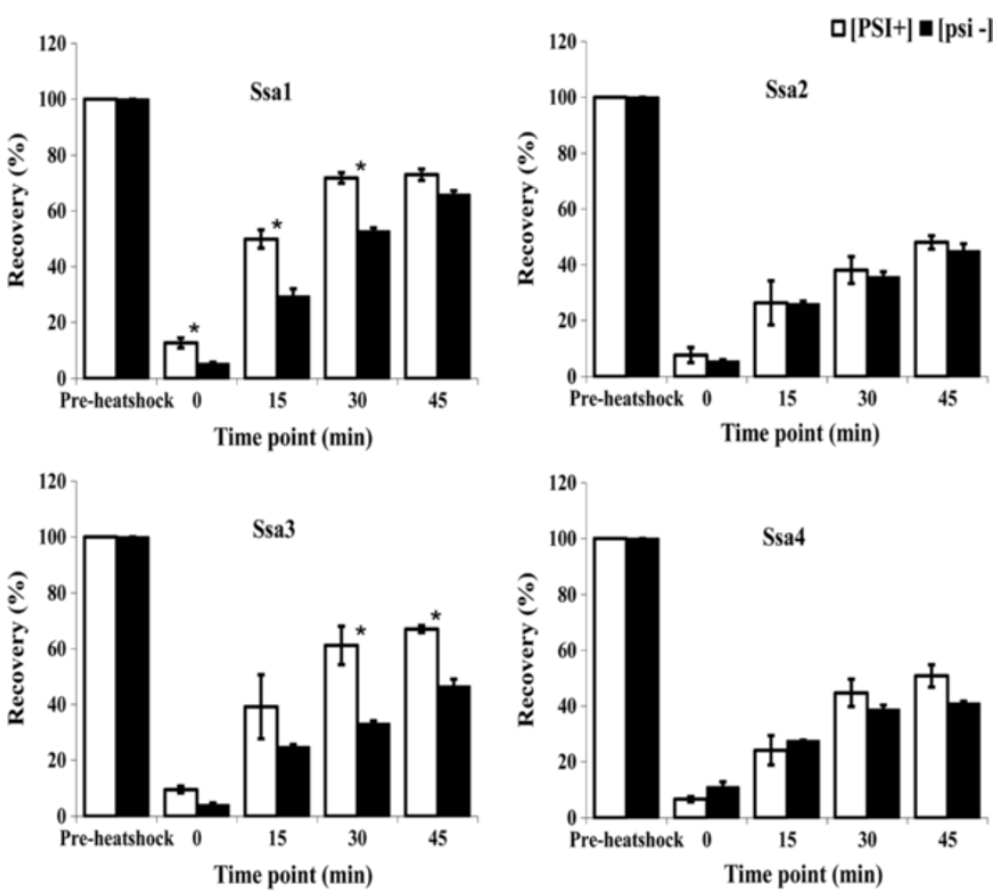

Figure 3 Comparison of luciferase activity of $\left[\mathrm{PSI}^{+}\right]$and $\left[\mathrm{psi}^{-}\right]$versions of the Ssa family. Overnight cultures were diluted in fresh SC medium lacking uracil to an $\mathrm{OD}_{600 \mathrm{~nm}}=0.1$. The cultures were then shifted to $37^{\circ} \mathrm{C}$ for 30 minutes to induce the expression of Hsp104. After 30 minutes at $37^{\circ} \mathrm{C}$, the cultures were shifted to $45^{\circ} \mathrm{C}$ for 1 hour. Cyclohexamide was added to the cultures after 50 minutes at $45^{\circ} \mathrm{C}$ to prevent any further synthesis of luciferase during the recovery period. Luciferase activity, expressed as a percentage of pre-heat shock activity, was measured at regular intervals during the recovery period of 45 minutes at $25^{\circ} \mathrm{C}$. White $\left[P S I^{+}\right]$, and black [psi $]$. (Bar indicates SEM. Value followed by * are significantly different at $\mathrm{p} \leq 0.05$. 
cells (Figure 4, bottom panels). Such an influence of $\left[\mathrm{PSI}^{+}\right]$ has been observed previously for the closely related G600 strain [51], although in the case of G402 the reason for this effect is unknown.

Similarly, we also see that cells expressing ether Ssa3 and $\mathrm{Ssa} 4$ exhibit greater resistance to $\mathrm{H}_{2} \mathrm{O}_{2}$ compared to Ssa1 and Ssa2 (Figure 5). Additionally, a prion effect on sensitivity is also observed, however this effect is only seen for $\mathrm{Ssa} 3$ with the $\left[\mathrm{PSI}^{+}\right]$variant being more resistant than $\left[p s i^{-}\right]$at $3 \mathrm{mM} \mathrm{H}_{2} \mathrm{O}_{2}$ and higher. These results are reproducible but the reasoning behind this sensitivity profile is not known.

\section{Global transcription profile of yeast strains expressing individual Ssa's}

Although the Hsp70-Ssa family carry out non-redundant cellular functions, increasing evidence suggests an array

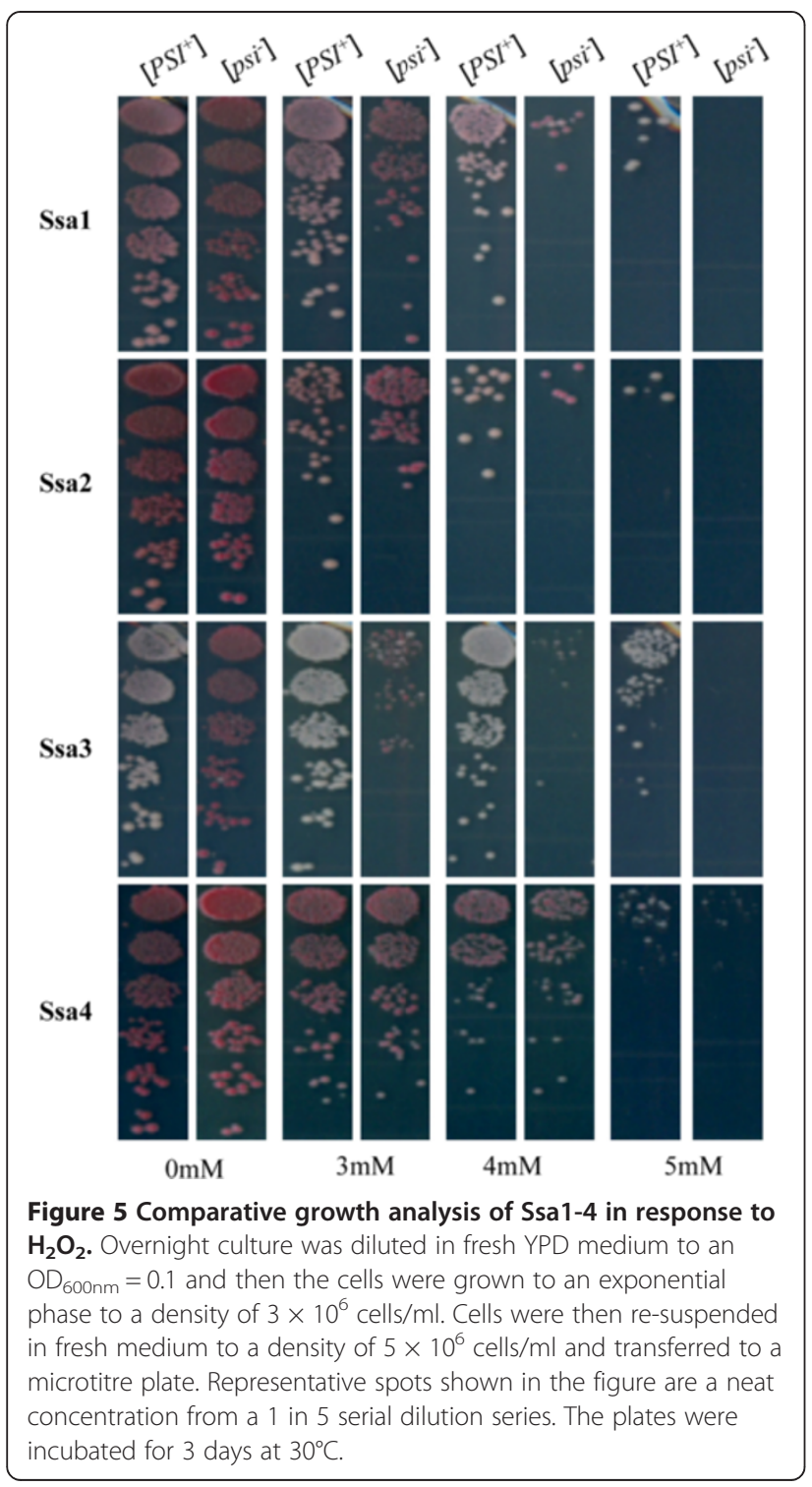

of Ssa family-specific functions exist. One such reason for the complex stress phenotypes exhibited in strains expressing individual Hsp70-Ssas could be due to changes in gene expression within the cell. We therefore assessed the global genome expression patterns for $\left[p s i^{-}\right]$ variants for cells expressing individual Hsp70-Ssa family members. We used cells expressing Ssa1 as the control comparison. To assess global gene expression we used the highly sensitive Yeast 3D-Gene Microarray platform (Toray Industries, Japan). We identified a total of 78, 134 and 298 genes induced (>2-fold induction) and 147, 120 and 426 genes repressed (>2-fold repression) when Ssa2, Ssa3 and Ssa4 were expressed respectively as a sole source of Ssa in the cells compared to Ssal (Figure 6 and Additional file 4: Table S2). The strain with Ssa4 generated the highest differential expression with 209 genes induced and 339 genes repressed. In contrast, strains expressing only Ssa2 or Ssa3 proteins showed a relatively smaller number of induced (16 and 47 respectively) and repressed (86 and 79 respectively) genes.

An alternative way of assessing the strains expressing each individual Hsp70-Ssa family member is in terms of the gene deletions. Effectively, cells expressing Ssa1 can be viewed as a $\Delta s s a 2 \Delta s s a 3 \Delta s s a 4$ deletion strain, cells expressing $\mathrm{Ssa} 2$ as a $\Delta s s a 1 \Delta s s a 3 \Delta s s a 4$ deletion strain, cells expressing $\mathrm{Ssa} 3$ as a $\Delta s s a 1 \Delta s s a 2 \Delta s s a 4$ and cells expressing $\mathrm{Ssa} 4$ as a $\Delta s s a 1 \Delta s s a 2 \Delta s s a 3$ deletion strain. By comparing and identifying the co-regulated genes shared amongst the Ssa1/2/3/4 classes we can infer the regulation patterns for single and double deletions of each Ssa family member from our data. The overlapping circles in the Venn diagram (Figure 6) shows the numbers of shared genes between each of the Ssa family members and the gene identities are shown in Additional file 4: Table S2. Microarray data for these experiments is publicly available through the NCBI's GEO database with accession number GSE32433.

RT-qPCR analysis was carried out to verify the microarray analysis. Twenty five genes were chosen based on their putative function and to represent a range of differential expression values among strains expressing Ssa1, Ssa2, Ssa3 or Ssa4 as the sole source of Ssa in the cells in a $\left[p i^{-}\right]$background. The expression levels were normalised with a housekeeping gene ACT1 and were LOG transformed. The comparative overview of the RT-qPCR and microarray results showed high degree of correlation and demonstrated the efficacy of both experiments (Figure 7 and Additional file 5: Table S3).

\section{Discussion}

Expressing each member of the cytosolic Ssa family of Hsp70 as the sole source of Ssa to study the effect on prion propagation reinforces that yeast prion propagation and maintenance are influenced to varying degrees 

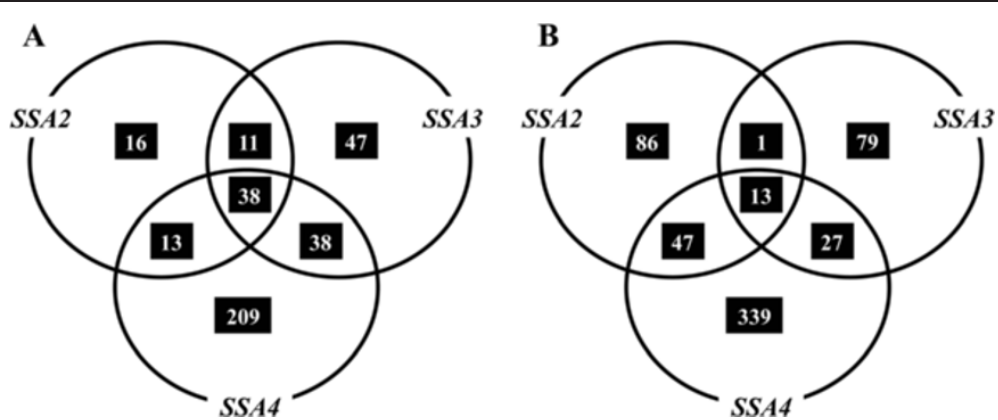

Figure 6 Comparative transcriptome profiling of the Ssa family. A Venn diagram representation of genes induced (A) or repressed (B) in different $\triangle$ ssa strains. Analysis was performed relative to expression levels of G402 expressing SSA1 as sole source of cytosolic Ssa protein.

by different members. $\left[\mathrm{PSI}^{+}\right]$is the infectious prion form of the Sup35 protein (eRF3) and $\left[P S I^{+}\right]$phenotype can be assessed by its ability to suppress the ade2-1 premature ochre mutation present within $A D E 2$ gene in strain G402. Sup35 protein (eRF3) under normal conditions in the cell encodes a translation release factor [52,53]. Aggregation of the Sup35 in $\left[\mathrm{PSI}^{+}\right]$cells causes nonsense suppression because of the depletion of Sup35 protein into an insoluble prion aggregate that is no longer functional. Thus, the strength of prion phenotype is directly proportional to the amount of Sup35 protein that has been depleted to insoluble prion aggregates [33]. Ssa family members differed in their abilities to propagate $\left[P S I^{+}\right]$, with Ssa3 being the most effective member in maintenance and propagation of $\left[\mathrm{PSI}^{+}\right]$, compared to other Ssa proteins. This result is consistent with a previous study by Sharma et al. [21]. Earlier studies by Schwimmer et al. and Roberts et al. [26,27] also found different effects of the Ssa family on [URE3] propagation. For example, overexpression of Ssa1 but not Ssa2 can cure [URE3], whereas a mutation in Ssa2 but not Ssa1 impairs [URE3] propagation. Recently, corresponding changes of a single ATPase domain residue (A83G in Ssa1, G83A in Ssa2) in Ssa1 or Ssa2 was able to allow



Figure 7 Comparative overview of microarray and RT-qPCR analysis. Twenty five genes were identified by microarray technique as being primed by yeast G402 strain carrying either Ssa1, 2, 3 or 4 as sole source of Ssa family protein. Both microarray and RT-qPCR analysis was conducted using total RNA extracted from 5-ml cultures of the [psi'] yeast strains carrying Ssa 1, 2, 3 or 4 grown overnight at $30^{\circ} \mathrm{C}$. The 3D-Gene ${ }^{\mathrm{TM}}$ Yeast Oligo chip S.cerevisiae $6 \mathrm{k}$ used for the microarray analysis according to the manufacturer 's instruction. Relative mRNA expression levels were quantified relative to that of the housekeeping gene $A C T 1$ (YFL039C) by $2^{\wedge-\Delta \Delta C t}$ method [43]. Bothe the global normalize value of microarray and the relative mRNA expression levels were LOG-transformed to linearize the data and the heat map of was created using TIGR MultiExperiment Viewer (MeV) [41]. Results are based on two experiments, each with three replicates. 
these Hsp70-Ssa family members to acquire the prion propagation behaviour of the other [28]. It appears that the evolution and occurrence of multiple Hsp70s within a species may be due to modified regulation of Hsp70 substrate-binding activity rather than changes to range of substrate that is recognised [28]. In S. cerevisiae, the activity of Ssa proteins is regulated by J-proteins [54] and NEFs such as Fes1 and Sse1/2 [55-60]. It has not been systematically tested whether the different Ssa proteins have different affinities for J-proteins and NEFs but remains an attractive possibility that this could partly explain different prion phenotypes when members of the Ssa family are individually expressed as a sole source of Ssa in the cell.

The Hsp104 protein influences cell survival under prolonged exposure to high temperatures [24]. At elevated temperatures, Hsp104 activity allows cells to survive by resolubilising heat-denatured proteins [34]. Apart from Hsp104, refolding of misfolded protein requires the activity of additional chaperone proteins such as Hsp70 and Hsp40. Thus Hsp104, in conjunction with Hsp70 and Hsp40, constitutes a protein disaggregation machinery leading to the resolubilisation of protein aggregates [61]. Both the constitutive and heat-inducible isoforms of Hsp70 functioned well with Hsp104 in refolding thermally denatured luciferase but preferentially, Ssa1 and Ssa3 were more active in vivo for luciferase refolding than Ssa2 and Ssa4 (Figure 3). Such differences may be reflected by the specific Hsp70 component of the protein disaggregation machinery. It has been proposed that heat inducible Hsp70s may function more efficiently with Hsp104 than constitutive Hsp70s [21]. However, this does not appear to be the case. Ssa3 clearly provides better levels of acquired thermotolerance than other Ssas (Figure 2), but even when cells are allowed to recover on medium that inhibits $\mathrm{Hsp} 104$ activity ( $\mathrm{GdnHCl}$ plates), cells expressing Ssa3 still recover remarkably well. This result suggests that for cells expressing $\mathrm{Ssa} 3$ as the sole Hsp70-Ssa, there is a greater impact of factors other than Hsp104 in determining levels of acquired thermotolerance. Additionally, the assessment of refolding activity of a model substrate such as luciferase does not necessarily correlate well with the in vivo refolding activity required for survival of heat stress.

Further complicating the interpretation of the luciferase refolding and acquired thermotolerance plate assays is the fact that Ssa1 is the most efficient in refolding denatured luciferase but does match or improve upon Ssa3 in the plate assay. Additionally, the presence of $\left[\mathrm{PSI}^{+}\right]$ caused a clear and reproducible increase in efficiency in luciferase refolding in the Ssa1 and Ssa3 expressing strains (Figure 3), which did not translate to increased survival in the acquired thermotolerance plate assays (Figure 2). Taken together these results also suggest a significant influence on levels of acquired thermotolerance from other cellular sources in addition to Hsp104.

Following further phenotypic stress analysis we also identified differences between the Ssas in response to oxidative and cell wall stress inducing agents (Figures 4 and 5). Of particular note is the trend for the inducible Ssas to provide better protection against both $\mathrm{H}_{2} \mathrm{O}_{2}$ and SDS. However, again a complex stress response is observed, as the presence of $\left[\mathrm{PSI}^{+}\right]$appears to influence sensitivity to SDS and to $\mathrm{H}_{2} \mathrm{O}_{2}$, particularly when only Ssa3 is expressed. The reason for this prion influence is not known, but has been observed before in the closely related strain G600 [51]. Given that cells expressing only Ssa3 have a much stronger prion phenotype (Figure 1b) then prion-specific effects may be expected in these cells compared to others expressing different Ssa family members.

The different and complex prion and stress phenotypes exhibited by cells expressing individual members of the Hsp70-Ssa family led us to hypothesize that in addition to any inherent functional differences that may exist amongst these highly homologous proteins, the potential existed for indirect phenotypic influences caused by changes in global gene expression. This hypothesis appears to hold true as under non-stress conditions and when expressed from the same promoter, there are many differences in the expression levels of a variety of genes between cells expressing different Hsp70-Ssa family members (Figure 6 and Additional file 4: Table S2). The changes in expression levels are in genes that are involved in a diverse set of cellular functions that would be predicted to influence a variety of cellular phenotypes (Additional file 4: Table S2). The full cellular implications for these changes in gene expression are difficult to interpret, but some inferences can be made in respect of the stress phenotypes we have observed in this study. For instance, cells expressing either $\mathrm{Ssa} 2$ or Ssa4 showed a 3-fold repression of CTA1. CTA1 encodes catalase A, which is involved in hydrogen peroxide detoxification in the peroxisomal and mitochondrial matrices [62,63]. Additionally, CTT1, a catalase that has a role in protection of cell from oxidative damage caused by hydrogen peroxide [64], was over 2-fold repressed in Ssa2. Repression of these genes could be an influencing factor in the comparative sensitivity to $\mathrm{H}_{2} \mathrm{O}_{2}$ of cells expressing solely Ssa2. Also, the transcriptional profiling of the Ssa family also revealed that the TRX2 gene, which confers resistance to $\mathrm{H}_{2} \mathrm{O}_{2}$ [65], was among the top listed induced genes in Ssa4 strain and this may provide an explanation for the comparative resistance to $\mathrm{H}_{2} \mathrm{O}_{2}$ displayed by cells expressing solely Ssa4. In addition to this, two other genes, GPX2, which is a glutathione peroxidise that is induced by oxidative stress [66] and GRX1, which is a glutaredoxin were induced (3.13 and 2.49 fold respectively) only in Ssa4 expressing cells. Glutaredoxins are the 
source primary defenses against mixed disulfides formed following oxidative damage to proteins [67].

Further linking phenotype to possible changes in gene expression, we observed changes in genes involved in cell wall integrity (CWI) signalling in Ssa3 expressing cells. The sensitivity of the $\left[\mathrm{psi}^{-}\right]$variant of $\mathrm{Ssa} 3$ to $0.005 \%$ SDS (cell wall damaging agent) compared to [ $\left.\mathrm{psi}^{-}\right]$variant of other Ssas, suggests a possible reduced efficiency in cell wall integrity signalling in the Ssa3 strain. The sensitivity displayed by cells expressing Ssa3 may be explained by the suppression of two genes, LRG1 and PMT6. LRG1 is a GTPase - activating protein (GAP) that is involved in the Pkc1-mediated signalling pathway that controls cell wall integrity [68], while PMT6 belongs to a family of protein mannosyltransferases that catalyses the transfer of mannose from dolichyl phosphate-D-mannose to protein serine/threonine residues of secretory proteins, a reaction essential for cell wall rigidity and cell integrity [69]. Both of these genes were repressed in only Ssa3 expressing cells by over 2fold (Additional file 4: Table S2). Our findings implicate a possible role for Hsp70-Ssa family in the CWI signalling pathway.

A previous study comparing the global gene expression changes in yeast upon mild heat shock to those for yeast cells deleted for both SSA1 and SSA2, identified differential expression of groups of genes that are very distinct from those identified in our analysis [70]. Significant differences in strain background and SSA deletion status exists between these studies and therefore a direct comparison between global gene expression data is not possible.

\section{Conclusion}

While it is clear that Hsp70-Ssa family members provide redundant functions within the yeast cell, it is also evident that within groups of highly homologous Hsp70s, family members may evolve to be much more efficient at aspects of these shared functions or have even developed new specific roles within the cell. However, a major conclusion from this study is that while some phenotypic differences observed between cells expressing different Hsp70-Ssa family members may result in part from intrinsic functional differences between Hsp70s, a significant contribution to strain phenotype may also be attributed to major changes in global gene expression within the cell.

\section{Availability of Supporting Data}

Microarray data is publicly available through the NCBI's Gene Expression Omnibus (GEO) database http://www. ncbi.nlm.nih.gov/geo/query/acc.cgi?acc=GSE32433.

\section{Additional files}

\begin{abstract}
Additional file 1: Table S1. Primers used for RT-qPCR.
Additional file 2: Figure S1. Expression of individual Hsp70-Ssa family members in yeast. To confirm expression of individual Ssa family members we used Western blotting with antibodies recognising Hsp70Ssa (top panel), Ssa1 or Ssa2 only and Ssa3 or Ssa4 only. We also assessed Hsp104 expression in these cells. Ssa1/2 and Ssa3/4 specific antibodies were a gift from Elizabeth Craig. Hsp104 antibody was a gift from John Glover. Loading control is membrane stained with amido black.
\end{abstract}

Additional file 3: Figure S2. Relative abundance of Hsp70 and Hsp104 in yeast cells expressing. individual Ssa's. Western blot analysis was performed to examine the abundance of Hsp70 and Hsp104. Blots probed with anti-Hsp70 antibodies (SPA822, Cambridge Biosciences) were stripped and re-probed with anti-Hsp104 antibodies (gift from John Glover). Membrane was then stained by amido black as a loading and transfer control, are shown (Load). The Ssa strains are indicated on the top.

Additional file 4: Table S2. Systematic and standard names and descriptions are based on Saccharomyces Genome Database (SGD) http:// www.yeastgenome.org; ${ }^{* *}$ Orange cells show the data more than 100.

Additional file 5: Table S3. Comparative overview of fold change for microarray and $\mathrm{QPCR}$ of 25 genes.

\section{Competing interests}

The authors declare that they have no competing interests.

\section{Authors' contributions}

GWJ conceived of the study, participated in its design and coordination and analysed the data. $\mathrm{NH}$ and SAC performed the genetic and phenotypic analysis. $\mathrm{NH}$ and SSA performed the qPCR analysis. DAF analysed the yeast expression data. NH, SSA and GWJ helped draft the manuscript. All authors read and approved the final manuscript.

\section{Acknowledgements}

We thank Daniel Masison for plasmids, Elizabeth Craig and John Glover for antibodies, used in this study. This work was supported in part by Science Foundation Ireland grant RFP/07/BIC493 awarded to GWJ. NH was supported by a John and Pat Hume postgraduate scholarship. Microarray analysis was performed by Toray Industries as part of a 3D-Gene ${ }^{\text {TM }}$ Competition Award to GWJ.

\section{Author details}

${ }^{1}$ Yeast Genetics Laboratory, Department of Biology, National University of Ireland Maynooth, Maynooth, County Kildare, Ireland. ${ }^{2}$ Section on Formation of RNA, National Institutes of Child Health and Human Development, National Institutes of Health, Bethesda, Maryland 20814, USA. ${ }^{3}$ ARS Sustainable Perennial Crops Laboratory, United States Department of Agriculture, Beltsville, Maryland, USA. ${ }^{4}$ Genome Evolution Laboratory, Department of Biology, National University of Ireland Maynooth, Maynooth, County Kildare, Ireland.

Received: 7 November 2013 Accepted: 7 March 2014

Published: 14 March 2014

\section{References}

1. Nollen EAA, Morimoto Rl: Chaperoning signaling pathways: molecular chaperones as stress-sensing heat shock proteins. J Cell Sci 2002, 115(14):2809-2816.

2. Sangster TA, Lindquist S, Queitsch C: Under cover: causes, effects and implications of Hsp90 - mediated genetic capacitance. Bioessays 2004, 26(4):348-362.

3. De Los RP, Ben-Zvi A, Slutsky O, Azem A, Goloubinoff P: Hsp70 chaperones accelerate protein translocation and the unfolding of stable protein aggregates by entropic pulling. Proc Natl Acad Sci USA 2006, 103(16):6166-6171.

4. Floer M, Bryant GO, Ptashne M: Hsp90/70 chaperones are required for rapid nucleosome removal upon induction of the GAL genes of yeast. Proc Natl Acad Sci USA 2008, 105(8):2975. 
5. Mayer M, Bukau B: Hsp70 chaperones: cellular functions and molecular mechanism. Cell Mol Life Sci 2005, 62(6):670-684.

6. Sharma D, Masison DC: Hsp70 structure, function, regulation and influence on yeast prions. Protein Pept. Lett. 2009, 16(6):571-581.

7. Daugaard $M$, Rohde $M$, Jäättelä $M$ : The heat shock protein 70 family: Highly homologous proteins with overlapping and distinct functions. FEBS letters 2007, 581(19):3702-3710.

8. Lindquist S, Craig E: The heat-shock proteins. Annu Rev Genet 1988, 22(1):631-677

9. Gupta RS, Singh B: Phylogenetic analysis of $70 \mathrm{kDa}$ heat shock protein sequences suggests a chimeric origin for the eukaryotic cell nucleus. Curr Biol 1994, 4(12):1104-1114.

10. Hunt C, Morimoto Rl: Conserved features of eukaryotic hsp70 genes revealed by comparison with the nucleotide sequence of human $h s p 70$. Proc Natl Acad Sci USA 1985, 82(19):6455.

11. Pelham $\mathrm{H}: \mathrm{Hsp} 70$ accelerates the recovery of nucleolar morphology after heat shock. EMBO J 1984, 3(13):3095.

12. Li GC, Li L, Liu YK, Mak JY, Chen L, Lee W: Thermal response of rat fibroblasts stably transfected with the human 70-kDa heat shock protein-encoding gene. Proc Natl Acad Sci USA 1991, 88(5):1681.

13. Li G, Li L, Liu R, Rehman M, Lee W: Protection from thermal stress by human hsp70 with or without its ATP-binding domain. Proc Natl Acad Sci USA 1992, 89:2036-2040.

14. Jäättelä M, Wissing D, Bauer PA, Li GC: Major heat shock protein hsp70 protects tumor cells from tumor necrosis factor cytotoxicity. EMBO 1992, 11(10):3507.

15. Tutar $Y$, Song $Y$, Masison DC: Primate chaperones Hsc70 (constitutive) and Hsp70 (induced) differ functionally in supporting growth and prion propagation in Saccharomyces cerevisiae. Genetics 2006, 172(2):851-861.

16. Gao B, Biosca J, Craig EA, Greene LE, Eisenberg E: Uncoating of coated vesicles by yeast hsp70 proteins. J Biol Chem 1991, 266(29):19565.

17. Kabani M, Martineau CN: Multiple hsp70 isoforms in the eukaryotic cytosol: mere redundancy or functional specificity? Curr Genomics 2008, 9(5):338

18. Werner-Washburne M, Stone DE, Craig EA: Complex interactions among members of an essential subfamily of hsp70 genes in Saccharomyces cerevisiae. Mol Cell Biol 1987, 7(7):2568-2577.

19. Boorstein WR, Ziegelhoffer T, Craig EA: Molecular evolution of the Hsp70 multigene family. J Mol Evol 1994, 38(1):1-17.

20. James P, Pfund C, Craig EA: Functional specificity among Hsp70 molecular chaperones. Science 1997, 275(5298):387-389.

21. Sharma D, Martineau CN, Le Dall MT, Reidy M, Masison DC, Kabani M: Function of SSA subfamily of Hsp70 within and across species varies widely in complementing Saccharomyces cerevisiae cell growth and prion propagation. PloS one 2009, 4(8):e6644.

22. Sharma D, Masison DC: Functionally redundant isoforms of a yeast $\mathrm{Hsp} 70$ chaperone subfamily have different antiprion effects. Genetics 2008, 179(3):1301-1311.

23. Perrett $\mathrm{S}$, Jones $\mathrm{GW}$ : Insights into the mechanism of prion propagation. Curr Opin Struct Biol 2008, 18(1):52-59.

24. Jones GW, Tuite MF: Chaperoning prions: the cellular machinery for propagating an infectious protein? Bioessays 2005, 27(8):823-832.

25. Reidy M, Masison DC: Modulation and elimination of yeast prions by protein chaperones and co-chaperones. Prion 2011, 5(4):245-249.

26. Schwimmer C, Masison DC: Antagonistic interactions between yeast $\left[\mathrm{PSI}^{+}\right]$ and [URE3] prions and curing of [URE3] by Hsp70 protein chaperone Ssa1p but not by Ssa2p. Mol Cell Biol 2002, 22(11):3590-3598.

27. Tibor Roberts B, Moriyama H, Wickner RB: [URE3] prion propagation is abolished by a mutation of the primary cytosolic Hsp70 of budding yeast. Yeast 2004, 21(2):107-117.

28. Sharma D, Masison DC: Single methyl group determines prion propagation and protein degradation activities of yeast heat shock protein Hsp70 chaperones Ssa1p and Ssa2p. Proc Natl Acad Sci USA 2011, 108(33):13665-13670.

29. Martineau CN, Beckerich JM, Kabani M: Flo11p-independent control of "mat" formation by hsp70 molecular chaperones and nucleotide exchange factors in yeast. Genetics 2007, 177(3):1679-1689.

30. Truman AW, Kristjansdottir K, Wolfgeher D, Hasin N, Polier S, Zhang H, Perrett S, Prodromou C, Jones GW, Kron SJ: CDK-dependent Hsp70 Phosphorylation controls G1 cyclin abundance and cell-cycle progression. Cell 2012, 151(6):1308-1318.
31. Jones GW, Masison DC: Saccharomyces cerevisiae Hsp70 mutations affect $\left[\mathrm{PSI}^{+}\right]$prion propagation and cell growth differently and implicate Hsp40 and tetratricopeptide repeat cochaperones in impairment of $\left[P S I^{+}\right]$. Genetics 2003, 163(2):495.

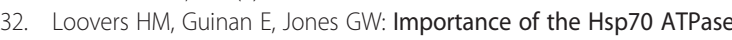
domain in yeast prion propagation. Genetics 2007, 175(2):621-630.

33. Jung $G$, Jones $G$, Wegrzyn RD, Masison DC: A Role for Cytosolic Hsp70 in Yeast $\left[\mathrm{PSI}^{+}\right]$Prion Propagation and $\left[\mathrm{PSI}^{+}\right]$as a Cellular Stress. Genetics 2000, 156:559-570.

34. Parsell DA, Kowal AS, Singer MA, Lindquist S: Protein disaggregation mediated by heat-shock protein Hspl04. Nature 1994, 372(6505):475-478

35. Sikorski RS, Hieter P: A system of shuttle vectors and yeast host strains designed for efficient manipulation of DNA in Saccharomyces cerevisiae. Genetics 1989, 122(1):19.

36. Liebman SW, Stewart JW, Sherman F: Serine substitutions caused by an ochre suppressor in yeast. J Mol Biol 1975, 94(4):595-610.

37. Cox BS: " $\psi$ " a cytoplasmic suppressor of super-suppressor in yeast. Heredity 1965, 20(121):505-521.

38. Jung G, Masison DC: Guanidine hydrochloride inhibits Hsp104 activity in vivo: a possible explanation for its effect in curing yeast prions. Curr Microbiol 2001, 43(1):7-10.

39. Moran C, Kinsella GK, Zhang ZR, Perrett S, Jones GW: Mutational Analysis of Sse1 (Hsp110) Suggests an Integral Role for this Chaperone in Yeast prion Propagation In Vivo. G3 2013, 3(8):1409-1418.

40. Saeed A, Sharov V, White J, Li J, Liang W, Bhagabati N, Braisted J, Klapa M, Currier T, Thiagarajan M: TM4: a free, open-source system for microarray data management and analysis. Biotechniques 2003, 34(2):374.

41. Ali SS, Nugent B, Mullins E, Doohan FM: Insights from the Fungus Fusarium oxysporum Point to High Affinity Glucose Transporters as Targets for Enhancing Ethanol Production from Lignocellulose. PloS one 2013, 8(1):e54701.

42. Livak KJ, Schmittgen TD: Analysis of Relative Gene Expression Data Using Real-Time Quantitative PCR and the $2^{-\Delta \Delta C T}$ Method. Methods 2001, 25(4):402-408

43. Ryan $T$, Joiner B: Normal probability plots and tests for normality. In Tech Rep. Penn State University Press: Dept. of Statistics. University Park; 1976.

44. Johnson NJ: Modified t tests and confidence intervals for asymmetrical populations. J Am Stat Soc 1978, 73(363):536-544.

45. Snedecor G, Cochran W: Statistical Methods. Ames: The lowa State University Press; 1980.

46. Tuite M, Mundy C, Cox B: Agents that cause a high frequency of genetic change from $\left[\mathrm{PSI}^{+}\right]$to $\left[\mathrm{psi}^{-}\right]$in Saccharomyces cerevisiae. Genetics 1981, 98(4):691-711

47. Bach S, Talarek N, Andrieu T, Vierfond J-M, Mettey Y, Galons H, Dormont D, Meijer L, Cullin C, Blondel M: Isolation of drugs active against mammalian prions using a yeast-based screening assay. Nat Biotechnol 2003, 21(9):1075-1081.

48. Tribouillard-Tanvier D, Béringue V, Desban N, Gug F, Bach S, Voisset C, Galons H, Laude H, Vilette D, Blondel M: Antihypertensive drug guanabenz is active in vivo against both yeast and mammalian prions. PloS one 2008, 3(4):e1981.

49. Landry J, Bernier D, Chrétien P, Nicole LM, Tanguay RM, Marceau N: Synthesis and degradation of heat shock proteins during development and decay of thermotolerance. Cancer Res 1982, 42(6):2457.

50. Ferreira PC, Ness F, Edwards SR, Cox BS, Tuite MF: The elimination of the yeast $\left[\mathrm{PSI}^{+}\right]$prion by guanidine hydrochloride is the result of Hsp104 inactivation. Mol Microbiol 2001, 40(6):1357-1369.

51. Fitzpatrick DA, O'Brien J, Moran C, Hasin N, Kenny E, Cormican P, Gates A, Morris DW, Jones GW: Assessment of Inactivating Stop Codon Mutations in Forty Saccharomyces cerevisiae Strains: Implications for $\left[\mathrm{PSI}{ }^{+}\right]$ Prion-Mediated Phenotypes. PloS one 2011, 6(12):e28684.

52. Stansfield I, Jones K, Kushnirov V, Dagkesamanskaya A, Poznyakovski A, Paushkin S, Nierras C, Cox B, Ter-Avanesyan M, Tuite M: The products of the SUP45 (eRF1) and SUP35 genes interact to mediate translation termination in Saccharomyces cerevisiae. EMBO J 1995, 14(17):4365.

53. Zhouravleva G, Frolova L, Le Goff X, Le Guellec R, Inge-Vechtomov S, Kisselev L, Philippe M: Termination of translation in eukaryotes is governed by two interacting polypeptide chain release factors, eRF1 and eRF3. EMBO J 1995 , 14(16):4065.

54. Sahi C, Craig EA: Network of general and specialty J protein chaperones of the yeast cytosol. Proc Natl Acad Sci USA 2007, 104(17):7163. 
55. Shaner L, Wegele H, Buchner J, Morano KA: The yeast Hsp110 Sse1 functionally interacts with the Hsp70 chaperones Ssa and Ssb. J Biol Chem 2005, 280(50):41262-41269.

56. Yam AYW, Albanèse V, Lin HTJ, Frydman J: Hsp110 cooperates with different cytosolic Hsp70 systems in a pathway for de novo folding. J Biol Chem 2005, 280(50):41252-41261.

57. Dragovic Z, Broadley SA, Shomura Y, Bracher A, Hartl FU: Molecular chaperones of the Hsp110 family act as nucleotide exchange factors of Hsp70s. EMBO J 2006, 25(11):2519-2528.

58. Raviol H, Sadlish H, Rodriguez F, Mayer MP, Bukau B: Chaperone network in the yeast cytosol: Hsp110 is revealed as an $\mathrm{Hsp} 70$ nucleotide exchange factor. EMBO J 2006, 25(11):2510-2518.

59. Kabani M, Beckerich JM, Brodsky JL: Nucleotide exchange factor for the yeast Hsp70 molecular chaperone Ssa1p. Mol Cell Biol 2002, 22(13):4677-4689.

60. Dragovic Z, Shomura Y, Tzvetkov N, Hartl FU, Bracher A: Fes1p acts as a nucleotide exchange factor for the ribosome-associated molecular chaperone Ssb1p. Biol Chem 2006, 387(12):1593.

61. Glover JR, Lindquist S: Hsp104, Hsp70, and Hsp40: A Novel Chaperone System that Rescues Previously Aggregated Proteins. Cell 1998, 94(1):73-82

62. Cohen G, Fessl F, Traczyk A, Rytka J, Ruis H: Isolation of the catalase A gene of Saccharomyces cerevisiae by complementation of the cta1 mutation. MGG 1985, 200(1):74-79.

63. Petrova V, Drescher D, Kujumdzieva A, Schmitt M: Dual targeting of yeast catalase A to peroxisomes and mitochondria. Biochem J 2004, 380:393-400.

64. Grant CM, Perrone G, Dawes IW: Glutathione and Catalase Provide Overlapping Defenses for Protection against Hydrogen Peroxide in the Yeast Saccharomyces cerevisiae. Biochem Biophys Res Commun 1998, 253(3):893-898.

65. Morano KA, Grant CM, Moye-Rowley WS: The response to heat shock and oxidative stress in Saccharomyces cerevisiae. Genetics 2012, 190(4):1157-1195.

66. Inoue Y, Matsuda T, Sugiyama K-i, Izawa S, Kimura A: Genetic analysis of glutathione peroxidase in oxidative stress response of Saccharomyces cerevisiae. J Biol Chem 1999, 274(38):27002-27009.

67. Luikenhuis S, Perrone G, Dawes IW, Grant CM: The yeast Saccharomyces cerevisiae contains two glutaredoxin genes that are required for protection against reactive oxygen species. Mol Biol Cell 1998, 9(5):1081-1091.

68. Lorberg A, Schmitz H-P, Jacoby J, Heinisch J: Lrg1p functions as a putative GTPase-activating protein in the Pkc1p-mediated cell integrity pathway in Saccharomyces cerevisiae. Mol. Genet. Genomics. 2001, 266(3):514-526.

69. Gentzsch M, Tanner W: The PMT gene family: protein O-glycosylation in Saccharomyces cerevisiae is vital. EMBO J 1996, 15(21):5752.

70. Matsumoto R, Akama K, Rakwal R, Iwahashi H: The stress response against denatured proteins in the deletion of cytosolic chaperones SSA1/2 is different from heat-shock response in Saccharomyces cerevisiae. BMC Genomics 2005, 6(1):141.

doi:10.1186/1471-2164-15-194

Cite this article as: Hasin et al:: Global transcript and phenotypic analysis of yeast cells expressing Ssa1, Ssa2, Ssa3 or Ssa4 as sole source of cytosolic Hsp70-Ssa chaperone activity. BMC Genomics 2014 15:194.

\section{Submit your next manuscript to BioMed Central and take full advantage of:}

- Convenient online submission

- Thorough peer review

- No space constraints or color figure charges

- Immediate publication on acceptance

- Inclusion in PubMed, CAS, Scopus and Google Scholar

- Research which is freely available for redistribution

Submit your manuscript at www.biomedcentral.com/submit 